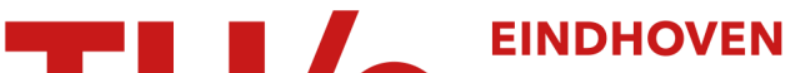 \\ UNIVERSITY OF \\ TECHNOLOGY
}

\section{Enforcing synchronization in oscillators with Huygens' coupling via feed-forward control}

Citation for published version (APA):

Pena Ramirez, J., \& Nijmeijer, H. (2019). Enforcing synchronization in oscillators with Huygens' coupling via feed-forward control. Nonlinear Dynamics, 98(4), 3009-3023. https://doi.org/10.1007/s11071-019-05062-x

\section{Document license:}

TAVERNE

DOI:

10.1007/s11071-019-05062-x

Document status and date:

Published: 01/12/2019

\section{Document Version:}

Publisher's PDF, also known as Version of Record (includes final page, issue and volume numbers)

\section{Please check the document version of this publication:}

- A submitted manuscript is the version of the article upon submission and before peer-review. There can be important differences between the submitted version and the official published version of record. People interested in the research are advised to contact the author for the final version of the publication, or visit the $\mathrm{DOI}$ to the publisher's website.

- The final author version and the galley proof are versions of the publication after peer review.

- The final published version features the final layout of the paper including the volume, issue and page numbers.

Link to publication

\section{General rights}

Copyright and moral rights for the publications made accessible in the public portal are retained by the authors and/or other copyright owners and it is a condition of accessing publications that users recognise and abide by the legal requirements associated with these rights.

- Users may download and print one copy of any publication from the public portal for the purpose of private study or research.

- You may not further distribute the material or use it for any profit-making activity or commercial gain

- You may freely distribute the URL identifying the publication in the public portal.

If the publication is distributed under the terms of Article 25fa of the Dutch Copyright Act, indicated by the "Taverne" license above, please follow below link for the End User Agreement:

www.tue.nl/taverne

Take down policy

If you believe that this document breaches copyright please contact us at:

openaccess@tue.nl

providing details and we will investigate your claim. 


\title{
Enforcing synchronization in oscillators with Huygens' coupling via feed-forward control
}

\author{
J. Pena Ramirez • H. Nijmeijer
}

Received: 21 December 2018 / Accepted: 11 June 2019 / Published online: 22 June 2019

(C) Springer Nature B.V. 2019

\begin{abstract}
This paper considers the problem of synchronizing a pair of oscillators with Huygens' coupling to a desired periodic trajectory, by using feedforward control. Two particular examples are considered: a modified version of the classical Huygens' system of pendulum clocks coupled through a common bar and a pair of self-sustained mass-springdamper oscillators mounted on a suspended bar. For both cases, it is demonstrated that if the coupling bar is excited by a suitably designed feed-forward control, then it is possible to indirectly force the oscillators to track a desired periodic trajectory. Additionally, sufficient and necessary conditions for the stability of the desired synchronous solution are derived by using the Poincaré method of perturbation and a numerical study is conducted in order to illustrate the theoretical findings. Ultimately, it is demonstrated that the proposed
\end{abstract}

J. Pena Ramirez $(\varangle)$

Center for Scientific Research and Higher Education at Ensenada, CONACYT-CICESE, Carretera

Ensenada-Tijuana 3918, Zona Playitas, C.P. 22860

Ensenada, B.C., Mexico

e-mail: jpena@cicese.mx

H. Nijmeijer

Department of Mechanical Engineering, Eindhoven

University of Technology, P.O. Box 513, $5600 \mathrm{MB}$

Eindhoven, The Netherlands

e-mail: h.nijmeijer@tue.nl feed-forward control induces the desired synchronous motion provided that, besides the coupling bar, there exists a diffusive coupling between the oscillators and the intrinsic parameters of the system satisfy certain constraints.

Keywords Synchronization · Huygens' coupling · Feed-forward control · Self-sustained oscillators

\section{Introduction}

\subsection{Historical notes}

One of the earliest reports on synchronization is due to the Dutch scientist Christiaan Huygens (1629-1695), who among other things, invented the pendulum clock and wrote one of the seminal scientific works of that times, namely the 'Horologium Oscillatorium' [1]. In late February 1665, Huygens, who was confined to his bedroom for several days due to an illness, made an exciting discovery: two of his pendulum clocks hanging from a common support - a wooden bar supported by two chairs-always swung together in opposite motion, i.e., the pendula were synchronized in anti-phase. In a letter to his father, Huygens referred to this odd phenomenon as 'the sympathy of two clocks' [2]. Nowadays, this sympathy is known as Huygens' synchronization [3-5] and the suspended bar coupling the clocks is referred to as Huygens' coupling [6,7]. 


\subsection{Literature review}

There exist several works aiming to provide a rigorous explanation for the synchronization phenomenon observed by Huygens in his pendulum clocks. For example, for the case of identical pendula, it has been found that the coupling strength, i.e., the ratio between the mass of the coupling bar and the mass of the pendula, plays a key role in the onset of synchronization and other behaviors like beating death, in which one of the clocks stop oscillating $[8,9]$.

Likewise, there are works, see, e.g., [10-14], in which it has been demonstrated that the damping in the system has a strong influence on the type of synchronization: for relatively large damping the pendula tend to synchronize in anti-phase, whereas for relatively small damping the pendula 'prefer' to synchronize in-phase.

On the other hand, the stiffness in the coupling bar also plays a key role in determining the frequency of the synchronous solution such that when the stiffness in the coupling is 'low' the oscillation frequency of the synchronous solution is larger than the average of eigenfrequencies of the uncoupled pendula, whereas when the stiffness is 'large' then the frequency of the synchronous solution is smaller than the average of the eigenfrequencies of the uncoupled oscillators $[15,16]$.

There also exist works where the pendula are assumed to be nonidentical [17-19]. In these cases, the pendula do not achieve complete synchronization, but rather frequency synchronization is observed.

Regarding the coupling bar, most of the models available in the literature consider that the bar is a rigid body, see, e.g., [8, 10,11,20,21]. Just recently, a model that (partly) takes into account the flexibility of the coupling bar has been derived [22].

At this point, it is worth mentioning that the case of more than two pendula coupled through a Huygens' coupling has also been the topic of current research. For example, in [23], a system consisting of two movable platforms with $N$ pendulum-like oscillators mounted on each platform is presented. The authors demonstrate that the pendula may exhibit the so-called chimera state: the pendula in one platform are completely synchronized, whereas those placed on the other platform oscillate out of synchrony.

Finally, it should be noted that different methods have been used to analyze the onset of synchronization in oscillators with Huygens' coupling. For exam- ple, in [8], conditions for the onset of synchronization in a simplified Huygens' system are derived by using a Poincaré map. Furthermore, in [24], the stability of the synchronous solutions is investigated using bifurcation analysis and the center manifold theory. Moreover, in $[21,25,26]$, a energy balance method is used for determining conditions for the onset of synchronous motion in coupled pendulum-like oscillators. And in [11], analytic conditions for the existence and stability of synchronous solutions in a pair of pendula with Huygens' coupling are provided. Likewise, several experimental platforms have been developed in order to further investigate Huygens' synchronization [8,10,2729], and recently, a modern version of Huygens' experiment has been created using monumental pendulum clocks [22].

\subsection{Problem statement}

Most of the works related to the study of Huygens' synchronization, including those mentioned in the previous subsection, consider the problem of selfsynchronization, i.e., the case where the pendula synchronize, in a natural way, either in-phase or in antiphase. Furthermore, in these studies the amplitude, frequency, and phase of the periodic synchronous solution are completely determined by the intrinsic parameters of the pendula and the coupling structure.

However, to the best of our knowledge, the case of controlled synchronization in Huygens' system of pendulum clocks has not been investigated in detail, although we have made some steps on this direction in our previous work, see [30]. In particular, the following question is still open: given the Huygens' system of pendulum clocks, how to enforce a desired synchronous periodic motion in the pendula?

This question becomes more relevant when the pendulum clocks are replaced by other self-sustained oscillators, like for example mechanical motors or electric generators, where besides synchrony, it is desired that the systems oscillate at certain frequency and phase.

Furthermore, in the case of self-synchronization, when the pendula synchronize in anti-phase, the coupling bar comes to standstill, whereas for the case that the pendula achieve in-phase synchronization, the coupling bar exhibits an oscillatory behavior. From an energy view point, the oscillation of the coupling for the in-phase synchronization regime may be an unde- 
sired situation because the energy required to keep the pendula synchronized in-phase is larger than in the case where the pendula achieve anti-phase synchronization. Hence, a natural question at this point is: how to control the coupling bar such that when the pendula synchronize in-phase the coupling bar does not oscillate but rather converges to standstill?

This work elaborates on these open questions. In particular, a suitable feed-forward control is designed, which 'destroys' the natural tendency of the pendula to synchronize and at the same time, the oscillation in the coupling bar are eliminated when the pendula achieve complete synchronization. Furthermore, since the feed-forward control converts the Huygens' system of coupled pendula into a nonautonomous system, the analysis techniques mentioned in Sect. 1.2 may not be useful in determining analytic conditions for the existence and stability of the desired synchronous solution. Consequently, in this work the analysis is conducted by using a perturbation method for nonautonomous systems.

\subsection{Contributions}

As mentioned before, most of the works reported in the literature about Huygens' synchronization consider the problem of self-synchronization, in which the synchronous solution in the system is entirely determined by the intrinsic properties of the oscillators. In contrast, this paper focuses on the case of controlled synchronization. Specifically, a feed-forward controller is designed, which allows inducing a desired synchronous motion in a pair of oscillators with Huygens' coupling. Furthermore, the proposed controller does not require to have any knowledge about the state variables of the oscillators. Instead, it only requires to have knowledge of the desired periodic trajectory and its first two derivatives. Therefore, the proposed synchronization scheme can be seen as an open-loop synchronization scheme. Note that the use of an open-loop synchronization scheme finds interesting applications like for example in the synchronization of power grids [31].

Also, there is a certain degree of novelty in the mathematical models considered here. In general, the classical Huygens' system of pendulum clocks is modeled by two simple pendula coupled through a rigid bar. Here, besides the coupling bar between the pendula, a spring has been added between the masses of the pendula.
This addition to the model is key in order to enforce the desired synchronous motion. In fact, in Sect. 3, it is analytically demonstrated that if this spring is removed then it is impossible to achieve the desired synchronous motion with the proposed feed-forward controller. The controller, however, has the limitation that it requires to impose constraints on the intrinsic parameters of the system.

Furthermore, the synchronization strategy discussed here may be applicable to a larger class of self-sustained oscillators. To illustrate this, Sect. 4 presents a 'generalized' Huygens' system, in which the pendulum clocks are replaced by self-sustained mass-springdamper oscillators. Also for this case, it is analytically and numerically demonstrated that the proposed controller induces the desired synchronous behavior in the oscillators.

In summary, this paper investigates the onset of controlled synchronization in pairs of self-sustained oscillators interacting via Huygens' coupling from a Control Theory perspective.

\subsection{Outline of the paper}

The rest of the manuscript is organized as follows. Section 2 introduces a key result for analyzing nonautonomous systems, which is based on the Poincaré method of perturbation. Next, Sects. 3 and 4 present the design of the feed-forward controller for achieving controlled synchronization in a pair of self-sustained oscillators with Huygens' coupling. Also, necessary and sufficient conditions for the stability of the desired synchronous solution are provided. As particular examples, we consider the classical Huygens' experiment of pendulum clocks, presented in Sect. 3, whereas in Sect. 4 a pair of self-sustained mass-spring-damper oscillators is considered. Then, in Sect. 5, the analytic results obtained in Sects. 3 and 4 are illustrated by means of numerical simulations. Finally, a discussion about the applicability of the results obtained here and some conclusions are presented in Sect. 6.

\section{Preliminaries: existence and stability of periodic solutions in nonautonomous systems}

Consider the nonautonomous system of equations

$\dot{y}_{s}=\lambda_{s} y_{s}+\mu F_{s}\left(y_{1}, \ldots, y_{l}, \mu, \omega t\right), \quad s=1, \ldots, l$, 
where $\lambda_{s}$ are the so-called characteristic exponents of system (1) when $\mu=0$ and $F_{S}$ are analytic and periodic functions of period $T=2 \pi / \omega$.

Assumption The characteristic exponents of (1) can be classified as follows:

$\lambda_{s}=i n_{s} \omega, \quad s=1, \ldots, k, \quad n_{s} \in \mathbb{Z}$,

$\lambda_{s}=-a_{s} \pm b_{s}, \quad s=k+1, \ldots, l, \quad a_{s}, b_{s} \in \mathbb{R}_{+}$.

Hence, for $\mu=0$, system (1) has the following asymptotic solutions

$y_{s}^{0}=\alpha_{s} e^{i n_{s} \omega t}, \quad s=1, \ldots, k$,

$y_{s}^{0}=0, \quad s=k+1, \ldots, l$,

where $\alpha_{s}$ are constants determining the amplitude of the solution.

The following theorem presented in [32] provides conditions for the existence and stability of periodic solutions in system (1). For the proof, the reader is referred to [33]. An analogous theorem for autonomous systems is provided in [34].

Theorem 1 Periodic solutions of the nonautonomous set of equations (1), which become periodic solutions (4) of the fundamental system, i.e., system (1) with $\mu=0$, can correspond only to such values of constants $\alpha_{1}, \ldots, \alpha_{k}$, which satisfy equations

$$
P_{s}\left(\alpha_{1}, \ldots, \alpha k\right):=\int_{0}^{T} F_{s}\left(y_{1}^{0}, \ldots, y_{l}^{0}, t\right) e^{-i n_{s} \omega t} d t=0,
$$

for $s=1, \ldots, k$. If for certain set of constants $\alpha_{1}=$ $\alpha_{1}^{*}, \ldots, \alpha_{k}=\alpha_{k}^{*}$ which satisfy condition (5), the real part of all roots $\chi$ of the following characteristic equation is negative

$$
p(\chi)=\operatorname{det}\left(\left.\frac{\partial Q}{\partial \alpha}\right|_{\alpha=\alpha^{*}}-\chi I\right)=0,
$$

where

$$
\left.\frac{\partial Q}{\partial \alpha}\right|_{\alpha=\alpha^{*}}=\left.\left[\begin{array}{ccc}
\frac{\partial P_{1}}{\partial \alpha_{1}} & \cdots & \frac{\partial P_{1}}{\partial \alpha_{k}} \\
\vdots & \ddots & \vdots \\
\frac{\partial P_{k}}{\partial \alpha_{1}} & \cdot & \frac{\partial P_{k}}{\partial \alpha_{k}}
\end{array}\right]\right|_{\alpha_{1}=\alpha_{1}^{*}, \ldots, \alpha_{k}=\alpha_{k}^{*}}
$$

where $I \in \mathbb{R}^{k \times k}$ is the identity matrix.

Then, for sufficiently small $\mu$, this set of constants will correspond to a unique, analytically w.r.t $\mu$ asymptotically stable periodic solution of Eq. (1). If the real part of at least one root of Eq. (6) is positive, then the corresponding solution is unstable.

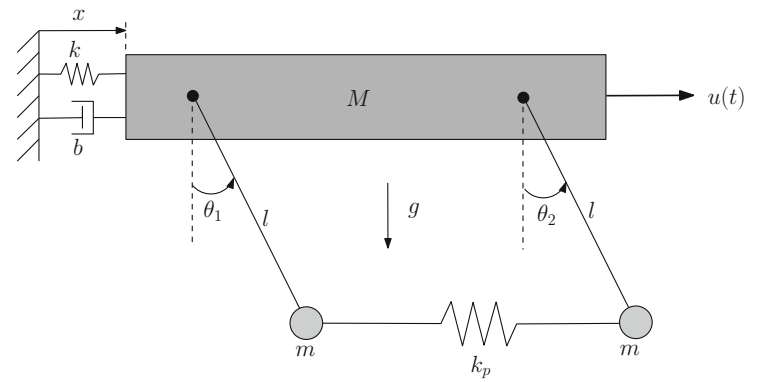

Fig. 1 Schematic diagram of the simplified Huygens' system

The following symbols are used through this work: $\mathbb{R}$ denotes the real numbers, the positive real numbers are indicated by $\mathbb{R}_{+}$, and $\mathbb{Z}$ is the set of integers.

\section{Controlled synchronization in a simplified Huygens' system using feed-forward control}

Consider the system depicted in Fig. 1. It consists of an actuated and rigid coupling bar, which is elastically attached to a fixed support by means of a spring and a damper. Attached to the bar, there are two self-sustained pendula, which are modeled as point masses attached to the lower end of a massless rod. Furthermore, note that the pendula are assumed to be identical.

The equations of motion describing the dynamic behavior of the system are

$$
\begin{aligned}
& m l^{2} \ddot{\theta}_{1}=-m l \ddot{x} \cos \theta_{1}-m g l \sin \theta_{1}-d \dot{\theta}_{1} \\
& \quad-k_{p} l^{2}\left(\sin \theta_{1}-\sin \theta_{2}\right) \cos \theta_{1}+\tau_{1}, \\
& m l^{2} \ddot{\theta}_{2}=-m l \ddot{x} \cos \theta_{2}-m g l \sin \theta_{2}-d \dot{\theta}_{2} \\
& \quad+k_{p} l^{2}\left(\sin \theta_{1}-\sin \theta_{2}\right) \cos \theta_{2}+\tau_{2}, \\
& (M+2 m) \ddot{x}=-k x-b \dot{x}-m l \sum_{i=1}^{2}\left(\ddot{\theta}_{i} \cos \theta_{i}\right. \\
& \left.-\dot{\theta}_{i}^{2} \sin \theta_{i}\right)+u(t),
\end{aligned}
$$

where $\theta_{i} \in \mathbb{R}$ in [rad] denotes the rotation angle of pendulum $i$, for $i=1,2, x \in \mathbb{R}$ is the horizontal displacement of the coupling bar in [m], the mass of each pendulum and the mass of the coupling bar are denoted by $m[\mathrm{~kg}]$ and $M[\mathrm{~kg}]$, respectively, $l[\mathrm{~m}]$ is the length of the massless rod of each pendulum, $d[\mathrm{~N} \mathrm{~ms} / \mathrm{rad}]$ is the damping constant corresponding to the rotational viscous damping in the pendula, $k[\mathrm{~N} / \mathrm{m}]$ and $b[\mathrm{~N} \mathrm{~s} / \mathrm{m}]$ are the stiffness and damping constants, respectively, of the coupling bar, and $g\left[\mathrm{~m} / \mathrm{s}^{2}\right]$ is the gravitational 
acceleration. The coupling bar is externally driven by the control input $u(t) \in \mathbb{R}$, which is to be designed, and the masses of the pendula are connected through a linear spring with elasticity $k_{p}[\mathrm{~N} / \mathrm{m}]$. Finally, each pendulum is converted into a self-sustained oscillator by the term $\tau_{i}$, which is given by

$\tau_{i}=v\left(\gamma^{2}-\theta_{i}^{2}\right) \dot{\theta}_{i}, \quad i=1,2$,

where $\gamma \in \mathbb{R}_{+}$defines the switching between positive and negative damping and $v \in \mathbb{R}_{+}$denotes the strength of the damping.

Remark 1 It should be noted that in the mechanical system depicted in Fig. 1 there are two types of coupling: at the top, the pendula are coupled by a rigid bar elastically attached to a support, i.e., by a Huygens' coupling, and in the bottom, the pendula are connected through a linear spring, i.e., a static coupling. In the upcoming analysis, it will become clear that the presence of the linear coupling spring is key for inducing a desired synchronous motion in the coupled pendula.

The (controlled) synchronization objective is to synchronize the pendula to a desired trajectory while preventing the oscillations in the coupling bar, i.e., it is desired that

$\lim _{t \rightarrow \infty} \theta_{i}(t)=\theta_{\text {ref }}(t)$,

$\lim _{t \rightarrow \infty} \dot{\theta}_{i}(t)=\dot{\theta}_{\text {ref }}(t), \quad \lim _{t \rightarrow \infty} x(t)=\dot{x}(t)=0$,

where $\theta_{\text {ref }} \in \mathbb{R}$ is a desired trajectory. In this work, it is assumed that $\theta_{\text {ref }}$ is the periodic signal

$\theta_{\text {ref }}(t)=\eta \sin \omega t$,

where the constants $\eta, \omega \in \mathbb{R}_{+}$are the amplitude and frequency, respectively, of the desired trajectory.

\subsection{Proposed feed-forward controller}

In order to achieve the desired synchronization objective (12), the following feed-forward control $u(t)$ is considered

$$
\begin{aligned}
u(t) & =2 m l \frac{\mathrm{d}^{2}}{\mathrm{~d} t^{2}} \sin \theta_{\text {ref }}(t) \\
& =2 m l\left(\ddot{\theta}_{\text {ref }} \cos \theta_{\text {ref }}-\dot{\theta}_{\text {ref }}^{2} \sin \theta_{\text {ref }}\right),
\end{aligned}
$$

where $m$ and $l$ are as described in (8)-(10) and $\theta_{\text {ref }}$ is as given in (13).
The design of the control input (14) is rather intuitive: it has been designed such that when the pendula synchronize to the desired reference $\theta_{\text {ref }}$, the term $-m l \sum_{i=1}^{2}\left(\ddot{\theta}_{i} \cos \theta_{i}-\dot{\theta}_{i}^{2} \sin \theta_{i}\right)+u(t)$ on the righthand side of Eq. (10) becomes zero and, as a consequence, the coupling bar, see Fig. 1, comes to standstill.

\subsection{Analysis of the controlled system}

Next, the controlled system (8)-(10), (14) is analyzed in order to determine conditions under which the control objective (12) is achieved.

As a first step, it is assumed that the desired angular displacement $\theta_{\text {ref }}$, see Eq. (13), is 'small', i.e., it is assumed that

- $[H-1]: \eta<<1$.

Consequently, system (8)-(10) with control (14) is (partly) linearized by replacing $\sin \theta_{i} \approx \theta_{i}, \cos \theta_{i} \approx 1$, and $\sin \theta_{\text {ref }} \approx \theta_{\text {ref }}, \cos \theta_{\text {ref }} \approx 1$. This yields

$$
\begin{aligned}
\ddot{\theta}_{1}= & -\frac{\ddot{x}}{l}-\frac{g}{l} \theta_{1}-\frac{d}{m l^{2}} \dot{\theta}_{1}-\frac{k_{p}}{m}\left(\theta_{1}-\theta_{2}\right)+\frac{\tau_{1}}{m l^{2}}, \\
\ddot{\theta}_{2}= & -\frac{\ddot{x}}{l}-\frac{g}{l} \theta_{2}-\frac{d}{m l^{2}} \dot{\theta}_{2}+\frac{k_{p}}{m}\left(\theta_{1}-\theta_{2}\right)+\frac{\tau_{2}}{m l^{2}}, \\
\ddot{x}= & -\frac{k}{M} x-\frac{b}{M} \dot{x} \\
& -\mu \sum_{i=1}^{2}\left(g \theta_{i}+l \dot{\theta}_{i}^{2} \theta_{i}+\frac{d}{m l} \dot{\theta}_{i}-\frac{\tau_{i}}{m l}\right)+\bar{u},
\end{aligned}
$$

where $\bar{u}=2 \mu l\left(\ddot{\theta}_{\text {ref }}-\dot{\theta}_{\text {ref }}^{2} \theta_{\text {ref }}\right)$ and $\mu:=\frac{m}{M}$ is the coupling strength. The next assumption is that the rotational damping in the pendula, the amplitude of the van der Pol term (11), and the stiffness coefficient $k_{p}$ of the coupling spring are 'small' in the sense that these terms scale with a small parameter $\mu$.

In other words, the following is assumed

- $[H-2]: d=\mu p, v=\mu c$, and $k_{p}=\mu \bar{k}_{p}$, with $p, c, \bar{k}_{p} \in \mathbb{R}_{+}$.

Another important assumption in the upcoming analysis is that the natural frequency of the uncoupled pendula, given by $\sqrt{g / l}$, coincides with the frequency $\omega$ of the desired reference signal $\theta_{\text {ref }}$ given in Eq. (13). Hence, it is assumed that the length of each pendulum satisfies 
- $[H-3]: l=\frac{g}{\omega^{2}}$.

Under assumptions [H-2] and $[H-3]$ and defining the dimensionless time variable $\tau=\omega t$ and dimensionless displacement variable $y=x / l$, it is possible to write system (15)-(17) in the following dimensionless form

$\frac{\mathrm{d}}{\mathrm{d} \tau}\left[\begin{array}{c}\theta_{1} \\ \dot{\theta}_{1} \\ \theta_{2} \\ \dot{\theta}_{2} \\ y \\ \dot{y}\end{array}\right]=\underbrace{\left[\begin{array}{cccccc}0 & 1 & 0 & 0 & 0 & 0 \\ -1 & 0 & 0 & 0 & q & s \\ 0 & 0 & 0 & 1 & 0 & 0 \\ 0 & 0 & -1 & 0 & q & s \\ 0 & 0 & 0 & 0 & 0 & 1 \\ 0 & 0 & 0 & 0 & -q & -s\end{array}\right]}_{A}\left[\begin{array}{c}\theta_{1} \\ \dot{\theta}_{1} \\ \theta_{2} \\ \dot{\theta}_{2} \\ y \\ \dot{y}\end{array}\right]+\mu f(\theta, t)$

where

$f(\theta, t)=\left[\begin{array}{c}0 \\ \frac{1}{m l^{2} \omega}\left(c\left(\gamma^{2}-\theta_{1}^{2}\right)-p\right) \dot{\theta}_{1}-\frac{\bar{k}_{p}}{m \omega^{2}}\left(\theta_{1}-\theta_{2}\right)-H\left(\theta_{1}, \theta_{2}, \dot{\theta}_{1}, \dot{\theta}_{2}, \bar{\theta}_{\mathrm{ref}}, \dot{\bar{\theta}}_{\mathrm{ref}}, \ddot{\bar{\theta}}_{\mathrm{ref}}\right) \\ \frac{1}{m l^{2} \omega}\left(c\left(\gamma^{2}-\theta_{2}^{2}\right)-p\right) \dot{\theta}_{2}+\frac{\bar{k}_{p}}{m \omega^{2}}\left(\theta_{1}-\theta_{2}\right)-H\left(\theta_{1}, \theta_{2}, \dot{\theta}_{1}, \dot{\theta}_{2}, \bar{\theta}_{\mathrm{ref}}, \dot{\bar{\theta}}_{\mathrm{ref}}, \ddot{\bar{\theta}}_{\mathrm{ref}}\right) \\ 0 \\ H\left(\theta_{1}, \theta_{2}, \dot{\theta}_{1}, \dot{\theta}_{2}, \bar{\theta}_{\mathrm{ref}}, \dot{\bar{\theta}}_{\mathrm{ref}}, \ddot{\bar{\theta}}_{\mathrm{ref}}\right)\end{array}\right]$,

where $H(\cdot)=\sum_{i=1}^{2} \theta_{i}\left(1+\dot{\theta}_{i}^{2}\right)+2\left(\ddot{\bar{\theta}}_{\text {ref }}-\dot{\bar{\theta}}_{\text {ref }}^{2} \bar{\theta}_{\text {ref }}\right)$.

Furthermore, it should be noted that the quadratic terms in $\mu$ have been neglected in Eq. (18). Moreover,

$q=\frac{k}{M \omega^{2}}, \quad$ and $\quad s=\frac{b}{M \omega}$,

and $\bar{\theta}_{\text {ref }}$ is the dimensionless reference signal, i.e., $\bar{\theta}_{\text {ref }}=\eta \sin (\tau)$ and $\dot{\bar{\theta}}_{\text {ref }}$ and $\ddot{\bar{\theta}}_{\text {ref }}$ are the first and second derivative of the dimensionless reference with respect to the dimensionless time $\tau$, i.e., $\dot{\bar{\theta}}_{\text {ref }}=\frac{\mathrm{d}}{\mathrm{d} \tau} \bar{\theta}_{\text {ref }}=$ $\eta \cos \tau$ and $\ddot{\bar{\theta}}_{\text {ref }}=\frac{\mathrm{d}^{2}}{\mathrm{~d} \tau^{2}} \bar{\theta}_{\text {ref }}=-\eta \sin \tau$.

The next step in the analysis is to diagonalize system (18). For this purpose, the following transformation is defined

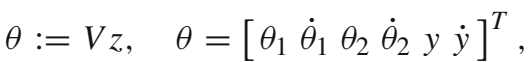

$z=\left[z_{1}, \ldots, z_{6}\right]^{T}, z_{1}, \ldots, z_{6} \in \mathbb{R}$,

where $V$ is the matrix of eigenvectors associated to matrix $A$ in Eq. (18). It is important to stress the fact that the algebraic multiplicity and the geometric multiplicity of the eigenvalues of matrix $A$ in Eq. (18) are equal and consequently, the columns of matrix $V$ are linearly independent, i.e., matrix $V$ is invertible.

Using transformation (21), system (18) is diagonalized as follows:

$\frac{\mathrm{d}}{\mathrm{d} \tau}\left[\begin{array}{c}z_{1} \\ z_{2} \\ z_{3} \\ z_{4} \\ z_{5} \\ z_{6}\end{array}\right]=\left[\begin{array}{cccccc}i & 0 & 0 & 0 & 0 & 0 \\ 0 & -i & 0 & 0 & 0 & 0 \\ 0 & 0 & i & 0 & 0 & 0 \\ 0 & 0 & 0 & -i & 0 & 0 \\ 0 & 0 & 0 & 0 & \sigma_{1} & 0 \\ 0 & 0 & 0 & 0 & 0 & \sigma_{2}\end{array}\right]\left[\begin{array}{c}z_{1} \\ z_{2} \\ z_{3} \\ z_{4} \\ z_{5} \\ z_{6}\end{array}\right]+\mu V^{-1} f(V z, t)$,

where $\sigma_{1}=-\frac{s}{2}+\sqrt{\left(s^{2}-4 q\right)}$ and $\sigma_{2}=-\frac{s}{2}-$ $\sqrt{\left(s^{2}-4 q\right)}$.

At this point, it is worth noting that system (22) has the form of the nonautonomous system (1), and therefore, system (22) can be analyzed by using the results presented in Sect. 2.

\subsection{Conditions for the existence of the desired synchronous solution}

Note that for $\mu=0$, system (22) has the following asymptotic solutions

$z_{1}(\tau)=\alpha e^{i \tau}, z_{2}=\alpha_{2} e^{-i \tau}, z_{3}(\tau)=\alpha_{3} e^{i \tau}$,

$z_{4}(\tau)=\alpha_{4} e^{-i \tau}, z_{5}(\tau)=z_{6}(\tau) \equiv 0$.

Next, in order to find the values of $\alpha$ 's in the above equation, it is necessary to consider Eq. (21) with $z_{i}$ 's as defined in (23). This yields the following expressions for the angular displacements

$\theta_{1}(\tau)=-i \alpha_{1} e^{i \tau}+i \alpha_{2} e^{-i \tau}$,

$\theta_{2}(\tau)=-i \alpha_{3} e^{i \tau}+i \alpha_{4} e^{-i \tau}$.

Furthermore, $\theta_{1}$ and $\theta_{2}$ must be real, and the amplitude should coincide with the desired amplitude $\eta$, see Eq. (13). Then, it follows that the values of $\alpha$ 's should be chosen as follows:

$\alpha_{1}=\alpha_{2}=\alpha_{3}=\alpha_{4}=\frac{\eta}{2}$.

Substitution of (26) into (24)-(25) yields the desired (dimensionless) synchronous solution 
$\theta_{1}(\tau)=\theta_{2}(\tau)=\eta \sin \tau$.

However, this solution exists only if condition (5) in Theorem 1 is satisfied. For the present case, this condition is given by

$$
\begin{aligned}
P_{1} & =P_{2}=P_{3}=P_{4} \\
& =-\frac{\pi \eta}{8 l^{2} m \omega}\left(c \eta^{2}+4\left(p-c \gamma^{2}\right)\right)=0 .
\end{aligned}
$$

Solving (28) for $\gamma$ yields

$\gamma= \pm \sqrt{\frac{\eta^{2} c+4 p}{4 c}}$.

Finally, note that this condition can be written in terms of the original parameters of the van der Pol term (11) as follows, see Assumption [H - 2] in previous subsection

$\gamma= \pm \sqrt{\frac{\eta^{2} v+4 d}{4 v}}$.

\subsection{Stability of the desired synchronous solution}

The stability of the desired (dimensionless) synchronous solution (27) can be determined from the characteristic equation (6) in Theorem 1 , which for the present case is given by

$p(\chi)=\chi^{4}+a_{3} \chi^{3}+a_{2} \chi^{2}+a_{1} \chi+a_{0}=0$,

where

$$
\begin{aligned}
a_{3}= & \frac{2 \pi s\left(\eta^{2}+2\right)}{\Gamma(q, s)}+\frac{\pi c \eta^{2}}{l^{2} m \omega}, \\
a_{2}= & \pi^{2}\left(4 \frac{\bar{k}_{p}^{2}}{m^{2} \omega^{4}}+\frac{3 \eta^{4}+16 \eta^{2}+16}{4 \Gamma(q, s)}\right. \\
& \left.+\frac{c^{2} \eta^{4}}{4 g l^{3} m^{2}}+\frac{c \eta^{2} s\left(s \eta^{2}+12\right)}{4 \omega l^{2} m \Gamma(q, s)}\right), \\
a_{1}= & \pi^{3}\left(\frac{8 \bar{k}_{p}^{2} s\left(\eta^{2}+2\right)}{m^{2} \omega^{4} \Gamma(q, s)}+\frac{c^{2} \eta^{4} s\left(\eta^{2}+4\right)}{8 g l^{3} m^{2} \Gamma(q, s)}\right. \\
& \left.+\frac{c \eta^{2}\left(3 \eta^{4}+16 \eta^{2}+16\right)}{8 \omega l^{2} m \Gamma(q, s)}+\frac{2 c \eta^{2} \bar{k}_{p}^{2}}{\omega l^{2} m^{3} \omega^{4}}\right) \\
a_{0}= & \frac{\pi^{4} \bar{k}_{p}^{2}}{m^{2} \omega^{4}}\left(\frac{l^{2} m\left(\eta^{2}+4\right)\left(3 \eta^{2}+4\right) \omega+c \eta^{2} s\left(\eta^{2}+4\right)}{l^{2} m \omega \Gamma(q, s)}\right),
\end{aligned}
$$

where $\Gamma(q, s)=q^{2}-2 q+1+s^{2}$.
This characteristic polynomial has roots with negative real parts if and only if

$$
\begin{aligned}
a_{n}>0, n & =0,1, \ldots, 3, \quad a_{3} a_{2}>a_{1}, \quad \text { and } \\
a_{3} a_{2} a_{1} & >a_{1}^{2}+a_{3}^{2} a_{0} .
\end{aligned}
$$

If these conditions are satisfied, the (dimensionless) desired synchronous solution (27) is asymptotically stable.

Remark 2 At this point, it is important to stress the fact that the coupling spring $k_{p}$ added to the Huygens' system, see Fig. 1, is an essential element for inducing the desired synchronous solution in the coupled pendula with the proposed open-loop control input (14). Note that if that spring is removed, then the coefficient $a_{0}$, see Eq. (35) of the characteristic polynomial (31) becomes 0 . As a consequence, one of the roots of (31) is zero, and therefore, the asymptotic stability properties of the desired synchronous solution are lost.

The above results are summarized in the following theorem.

Theorem 2 Consider the simplified Huygens' system of coupled pendula given in Eqs. (8)-(10) and assume that it is desired to achieve the synchronization objective (13). Furthermore, assume that the amplitude $\eta$ of the desired trajectory (13) is 'small' $(\eta<<1)$ and the intrinsic parameters $l$ and $\gamma$ of the self-sustained pendula satisfy

$l=\frac{g}{\omega^{2}}, \quad$ and $\quad \gamma=\sqrt{\frac{\eta^{2} v+4 d}{4 v}}$.

Then, the feed-forward control

$$
\begin{aligned}
u(t) & =2 m l \frac{d^{2}}{d t^{2}} \sin \theta_{\text {ref }}(t) \\
& =2 m l\left(\ddot{\theta}_{\text {ref }} \cos \theta_{\text {ref }}-\dot{\theta}_{\text {ref }}^{2} \sin \theta_{\text {ref }}\right),
\end{aligned}
$$

enforces the synchronization objective given in Eq. (12). Furthermore, if conditions (36) are satisfied, then the synchronous solution (12) is locally asymptotically stable.

\section{Controlled synchronization in a generalized Huygens' system using feed-forward control}

The previous section has shown that the proposed feedforward control can induce the desired synchronous 


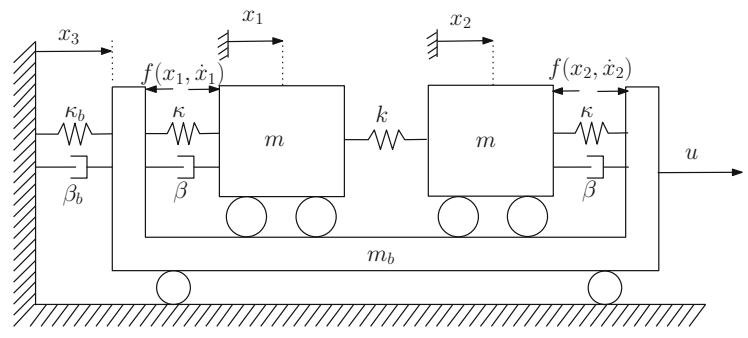

Fig. 2 Schematic diagram of the generalized Huygens' system

motion in a pair of pendula with Huygens' coupling. However, the synchronization strategy proposed here does not restrict to only pendula, but it may be applicable to other self-sustained oscillators. To demonstrate this claim, this section presents an study related to the onset of controlled synchronization in a generalized Huygens' system, in which the pendulum clocks have been replaced by two self-sustained mass-springdamper oscillators, cf. [35].

The schematic diagram of the system is depicted in Fig. 2. It consists of two oscillators of mass $m[\mathrm{~kg}]$, attached to a moving bar by means of a spring with stiffness $\kappa \in \mathbb{R}_{+}[\mathrm{N} / \mathrm{m}]$ and a damper with damping coefficient $\beta \in \mathbb{R}_{+}[\mathrm{N} \mathrm{s} / \mathrm{m}]$. The term $f\left(x_{i}, \dot{x}_{i}\right), i=1,2$ is a nonlinear function which converts oscillator $i$ into a self-sustained oscillator. On the other hand, the coupling bar has mass $m_{b}[\mathrm{~kg}]$ and it is attached to a fixed support by a spring and a damper with linear stiffness and damping characteristic denoted by $\kappa_{b} \in \mathbb{R}_{+}[\mathrm{N} / \mathrm{m}]$ and $\beta_{b} \in \mathbb{R}_{+}[\mathrm{N} \mathrm{s} / \mathrm{m}]$, respectively. Furthermore, the oscillators are also coupled by a spring with stiffness $k$ $[\mathrm{N} / \mathrm{m}]$ and the coupling bar can be externally actuated with the control input $u[\mathrm{~N} / \mathrm{kg}]$. Finally, the absolute displacements of both oscillators and the coupling bar are denoted by $x_{i} \in \mathbb{R}[\mathrm{m}], i=1,2,3$, respectively.

The mathematical model describing the dynamic behavior of the system depicted in Fig. 2 is given by

$$
\begin{aligned}
\ddot{x}_{1}= & -\omega^{2}\left(x_{1}-x_{3}\right)-2 \xi \omega\left(\dot{x}_{1}-\dot{x}_{3}\right) \\
& -k\left(x_{1}-x_{2}\right)+f\left(x_{1}, \dot{x}_{1}\right), \\
\ddot{x}_{2}= & -\omega^{2}\left(x_{2}-x_{3}\right)-2 \xi \omega\left(\dot{x}_{2}-\dot{x}_{3}\right) \\
& -k\left(x_{2}-x_{1}\right)+f\left(x_{2}, \dot{x}_{2}\right), \\
\ddot{x}_{3}= & -w_{b}^{2} x_{3}-2 \xi_{b} w_{b} \dot{x}_{3}-\mu \sum_{i=1}^{2} \ddot{x}_{i}+u,
\end{aligned}
$$

where $\mu=\frac{m}{m_{b}}$ is the coupling strength, $\omega=\sqrt{\frac{\kappa}{m}} \in \mathbb{R}_{+}$ and $\xi=\frac{\beta}{2 \omega} \in \mathbb{R}_{+}$is the eigenfrequency (in rad) and the dimensionless damping coefficient of the oscillators, respectively. Similarly, $\omega_{b}=\sqrt{\frac{\kappa_{b}}{m_{b}}} \in \mathbb{R}_{+}$and $\xi_{b}=\frac{\beta_{b}}{2 \omega_{b}}$ denote the eigenfrequency (in rad) and the dimensionless damping in the coupling bar.

On the other hand, the nonlinear functions $f\left(x_{i}, \dot{x}_{i}\right)$ are

$f\left(x_{i}, \dot{x}_{i}\right)=-\lambda\left(H_{i}\left(x_{i}, \dot{x}_{i}\right)-H^{*}\right) \dot{x}_{i}, \quad i=1,2$,

where $\lambda, H^{*} \in \mathbb{R}_{+}$are design parameters determining the amplitude of the self-sustained oscillation, and function $H_{i}$ is the Hamiltonian corresponding to the uncoupled oscillator $i$ as is given by

$H_{i}=\frac{1}{2} m \dot{x}_{i}^{2}+\frac{1}{2} \kappa x_{i}^{2}, \quad i=1,2$.

\subsection{Feed-forward controller}

Similar to the case of coupled pendula discussed in previous section, here the control objective is to synchronize the oscillators to a desired periodic trajectory $x_{\text {ref }}(t)$ and to control the vibrations in the coupling bar. In other words, the synchronization control objective is

$\lim _{t \rightarrow \infty} x_{1}(t)=x_{2}(t)=x_{\text {ref }}(t)$, and $\lim _{t \rightarrow \infty} x_{3}=0$.

For simplicity in the upcoming analysis, we consider the following periodic reference

$x_{\mathrm{ref}}(t)=\eta \sin \left(\omega_{d} t\right)$,

where $\eta \in \mathbb{R}_{+}$is the amplitude and $\omega_{d} \in \mathbb{R}_{+}$is the desired oscillation frequency.

On the other hand, similar to Eq. (14), the following feed-forward controller is considered

$u(t)=2 \mu \frac{\mathrm{d}^{2}}{\mathrm{~d} t^{2}} x_{r e f(t)}=-2 \mu \eta \omega_{d}^{2} \sin \left(\omega_{d} t\right)$,

\subsection{Existence and stability conditions for the synchronous solution}

Now the controlled system is analyzed by using the mathematical tool presented in Sect. 2. Specifically, in this part we derive necessary and sufficient conditions, which guarantee the existence and local asymptotic stability of the desired synchronous solution. 
First, by rescaling the time by $\tau=\omega t$ system (39) is written in the dimensionless form

$$
\begin{aligned}
x_{i}^{\prime \prime}= & -\left(x_{i}-x_{3}\right)-p\left(x_{i}^{\prime}-x_{3}^{\prime}\right) \\
& -\bar{\lambda}\left(a x_{i}^{\prime 2}+\kappa x_{i}^{2}-\gamma\right) x_{i}^{\prime},-k\left(x_{i}-x_{j}\right), \\
x_{3}^{\prime \prime}= & -q x_{3}-s x_{3}^{\prime}-\mu \sum_{i=1}^{2} x_{i}^{\prime \prime}-\frac{2 \mu \eta \omega_{d}^{2}}{\omega^{2}} \sin \tau,
\end{aligned}
$$

for $i, j=1,2$, and the primes denote differentiation with respect to the dimensionless time $\tau$, and

$$
\begin{aligned}
& p=2 \xi, \quad \bar{\lambda}=\frac{\lambda}{2 \omega}, \quad a=m \omega^{2}, \quad \gamma=2 H^{*}, \\
& q=\frac{\omega_{b}^{2}}{\omega^{2}}, \quad \text { and } \quad s=\frac{2 \xi_{b} \omega_{b}}{\omega} .
\end{aligned}
$$

Next, the following assumptions are considered:

- $[H-4]$ : The diffusive coupling $k$, the damping $p$ in the oscillators, and the amplitude of the nonlinearity $\bar{\lambda}$ are small, i.e., $k=\mu \bar{k}, p:=\mu d$, and $\bar{\lambda}:=\mu \alpha$, with $d, \alpha \in \mathbb{R}_{+}$.

- $[H-5]$ : The natural frequency $\omega$ of the uncoupled oscillators coincides with the frequency $\omega_{d}$ of the desired reference signal $\theta_{\text {ref }}$ given in Eq. (43).

Note that assumption [ $H-5]$ can always be satisfied if the stiffness coefficient of the oscillators is chosen as $\kappa=m \omega_{d}^{2}$ or if the mass of the oscillators is chosen as $m=\frac{\kappa}{\omega_{d}^{2}}$.

Under these assumptions and by neglecting quadratic terms in $\mu$, system (45)-(46) can be written in the weakly nonlinear form

$x^{\prime}=A x+\mu \Phi(x)$,

where $x=\left[\begin{array}{llllll}x_{1} & x_{1}^{\prime} & x_{2} & x_{2}^{\prime} & x_{3} & x_{3}^{\prime}\end{array}\right]^{T}$ and

$$
\begin{aligned}
& A=\left[\begin{array}{cccccc}
0 & 1 & 0 & 0 & 0 & 0 \\
-1 & 0 & 0 & 0 & 1 & 0 \\
0 & 0 & 0 & 1 & 0 & 0 \\
0 & 0 & -1 & 0 & 1 & 0 \\
0 & 0 & 0 & 0 & 0 & 1 \\
0 & 0 & 0 & 0 & -q & -s
\end{array}\right], \\
& \Phi(x)=\left[\begin{array}{c}
0 \\
-\alpha\left(a x_{1}^{\prime 2}+\kappa x_{1}^{2}-\gamma\right) x_{1}^{\prime}-d\left(x_{1}^{\prime}-x_{3}^{\prime}\right)-\bar{k}\left(x_{1}-x_{2}\right) \\
0 \\
-\alpha\left(a x_{2}^{\prime 2}+\kappa x_{2}^{2}-\gamma\right) x_{2}^{\prime}-d\left(x_{2}^{\prime}-x_{3}^{\prime}\right)-\bar{k}\left(x_{2}-x_{1}\right) \\
0 \\
x_{1}+x_{2}-2 x_{3}
\end{array}\right] .
\end{aligned}
$$

Furthermore, system (48)-(50) is diagonalized by defining the transformation

$x=V z$, where $z=\left[\begin{array}{llllll}z_{1} & z_{2} & z_{3} & z_{4} & z_{5} & z_{6}\end{array}\right]^{T}$ and $V$ is the matrix of eigenvectors corresponding to matrix $A$ given in (49). Then, by applying this transformation to system (48) yields

$z^{\prime}=D z+\mu V^{-1} \Phi(V z)$,

where $D$ is a diagonal matrix containing the eigenvectors of matrix $A$, i.e.,

$D=\operatorname{diag}\left(i,-i, i,-i, \sigma_{1}, \sigma_{2}\right)$,

where $\sigma_{1}=\frac{1}{2}\left(-s-\sqrt{\left(s^{2}-4 q\right)}\right)$ and $\sigma_{2}=\frac{1}{2}(-s+$ $\left.\sqrt{\left(s^{2}-4 q\right)}\right)$.

Hence, for $\mu=0$, system (52) has the following asymptotic solutions

$z_{1}(\tau)=r_{1} e^{i(\tau)}, \quad z_{2}(\tau)=r_{2} e^{-i(\tau)}$,

$z_{3}(\tau)=r_{3} e^{i(\tau)}, \quad z_{4}(\tau)=r_{4} e^{-i(\tau)}$,

and $z_{5}(\tau)=z_{6}(\tau)=0$. The coefficients $r_{1}$ to $r_{4}$ are arbitrary parameters determined by the initial conditions.

Note that (54) is indeed a complete family of possible solutions. However, remember that we are interested in only one solution, namely the solution for which system (48) converges to the (dimensionless) synchronous solution $x_{\text {ref }}(\tau)=\eta \sin \tau$. To find the values of $r_{j}, j=1, \ldots, 4$, we use transformation (51) with $z_{l}, l=1, \ldots, 6$ as defined in (54). This yields

$x_{1}=-r_{1} e^{i \tau} i+r_{2} e^{-i \tau} i, \quad x_{1}^{\prime}=r_{1} e^{i \tau}+r_{2} e^{-i \tau}$,

$x_{2}=-r_{3} e^{i \tau} i+r_{4} e^{-i \tau} i, \quad x_{2}^{\prime}=r_{3} e^{i \tau}+r_{4} e^{-i \tau}$,

$x_{3}=x_{3}^{\prime}=0$.

Hence, in order to have the desired synchronous solution $x_{1}=x_{2}=\eta \sin \tau$, the values of $r_{j}, j=$ $1, \ldots, 4$, should satisfy

$r_{1}=r_{2}=r_{3}=r_{4}=\frac{\eta}{2}$.

Next, Theorem 1 is used in order to determine if the desired solution (54)-(58) exists and is locally asymptotically stable. In this case, conditions (5) in Theorem 1 are simply given by 


$$
\begin{aligned}
P_{1} & =P_{2}=P_{3}=P_{4} \\
& =-\frac{\pi \eta}{8}\left(4 d-4 \alpha \gamma+3 a \alpha \eta^{2}+\alpha \eta^{2} k\right)=0 .
\end{aligned}
$$

Solving condition (59) for parameter $\gamma$ yields

$\gamma=\frac{4 d+(3 a+k) \alpha \eta^{2}}{4 \alpha}$.

From Theorem 1 it follows that condition (60) is a necessary and sufficient condition for the existence of the desired synchronous solution $x_{1}(\tau)=x_{2}(\tau)=$ $\eta \sin \tau$ in system (48).

Remark 3 Note that by using (47) and Assumption [H4], condition (60) can be expressed in terms of the original parameters of system. This yields the condition

$H^{*}=\frac{16 \xi \omega+\left(3 m \omega^{2}+\kappa\right) \lambda \eta^{2}}{8 \lambda}$.

On the other hand, the local asymptotic stability of the desired solution (54)-(58) can be determined from Eq. (6) in Theorem 1. This yields the following characteristic polynomial

$p(\chi):=\chi^{4}+a_{3} \chi^{3}+a_{2} \chi^{2}+a_{1} \chi+a_{0}=0$,

where

$a_{3}=\left(\frac{4 s}{\Gamma(q, s)}+\alpha \eta^{2}(3 a+\kappa)\right) \pi$,
$a_{2}=\left(4 \bar{k}^{2}+\frac{\alpha^{2} \eta^{4}(3 a+\kappa)^{2}}{4}+\frac{9 a \alpha \eta^{2} s+3 \alpha \eta^{2} \kappa s+4}{\Gamma(q, s)}\right) \pi^{2}$,

$a_{1}=\left(2 \bar{k}^{2}(3 a+\kappa) \alpha \eta^{2}\right.$ $\left.+\frac{32 s \bar{k}^{2}+(3 a+\kappa) \alpha \eta^{2}\left((3 a+\kappa) \alpha \eta^{2} s+4\right)}{2 \Gamma(q, s)}\right) \pi^{3}$,

$a_{0}=\left(\frac{4 \bar{k}^{2}\left((3 a+\kappa) \alpha \eta^{2} s+4\right)}{\Gamma(q, s)}\right) \pi^{4}$,

where $\Gamma(q, s)$ is as defined in Eqs. (32)-(35).

Finally, if coefficients (63) to (66) satisfy conditions (36) then the synchronous solution $x_{1}(\tau)=x_{2}(\tau)=$ $\eta \sin \tau$ in system (48) is locally asymptotically stable.

Remark 4 Again, note that the spring $k=\mu \bar{k}$, which couples the oscillators, see Fig. 2, is essential for the stability of the desired synchronous solution. Specifically, note that if this spring is removed, i.e., if $\bar{k}=0$, then $a_{0}=0$ in Eq. (66), and consequently, one of the roots of (62) is equal to zero and the synchronous solution is not asymptotically stable.
It is important to note that the above results are directly applicable to the weakly nonlinear system (48), in which quadratic terms in $\mu$ have been neglected. However, under suitable assumptions, these results are also applicable to the original system (39). In fact, using the above results and this discussion yields to the following result for the original system (39).

Theorem 3 Consider the mechanical system consisting of two self-sustained oscillators with Huygens' coupling, which is described by model (39) with 'small' damping, 'small' nonlinearity (40), and coupling spring with positive stiffness $k$. Furthermore, assume that the coupling bar is driven by the feedforward control u given in (44).

Then, the synchronous solution (42) exists and is locally asymptotically stable if and only if the following is satisfied: (1) the eigenfrequency $\omega$ of the uncoupled oscillators coincides with the frequency $\omega_{d}$ of the desired synchronous solution, (2) the term $H^{*}$ in (41) satisfies condition (61), and (3) the roots of the characteristic polynomial (62) have negative real parts.

\section{Numerical results}

In this section, the analytic results obtained in the previous section are illustrated by means of computer simulations.

\subsection{Huygens' system of pendulum clocks with feed-forward control}

First, system (8)-(10) is considered with the following parameter values cf. [36]: $m=0.1[\mathrm{~kg}], k=1[\mathrm{~N} / \mathrm{m}]$, $b=0.5[\mathrm{~N} \mathrm{~s} / \mathrm{m}], d=0.001[\mathrm{~N} \mathrm{~ms} / \mathrm{rad}], g=9.81$ $\left[\mathrm{m} / \mathrm{s}^{2}\right], k_{p}=1[\mathrm{~N} / \mathrm{m}]$, and $v$ in Eq. (11) is $v=0.57$ $\left[\mathrm{kgm}^{2} / \mathrm{rad}^{3} \mathrm{~s}\right]$. Furthermore, the reference signal is as given in (13) with $\eta=0.05$ [-] and $\omega=8.8589$ [rad]. For these parameter values, it follows from Theorem 2 that the length $l$ of each pendulum and the term $\gamma$ of the van der Pol term (11) should be chosen as follows: $l=0.125[\mathrm{~m}]$ and $\gamma=0.0488$ [rad]. Moreover, for the considered parameter values, the roots of the characteristic polynomial (31) are: $\chi_{1}=-1.213+120.1 i$, $\chi_{2}=-1.213-120.1 i, \chi_{3}=-3.187+5.99 i$, and $\chi_{4}=-3.187-5.99 i$. Consequently, all conditions of Theorem 2 are satisfied and it is expected that the 


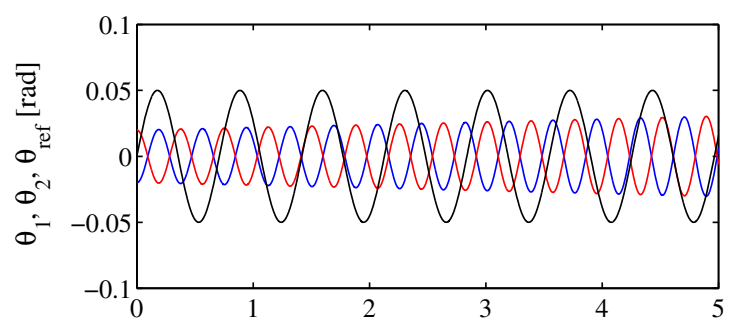

(a)

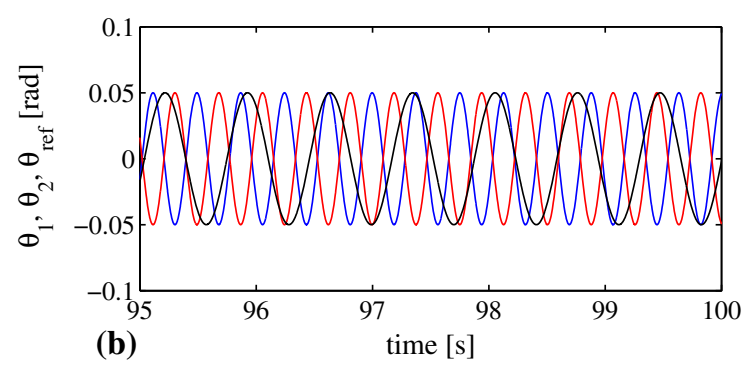

Fig. 3 Limit behavior of the uncontrolled system (8)-(10). Top: transient behavior. Bottom: 'steady' behavior. In both plots, the colors indicate the following. Blue: $\theta_{1}$, red: $\theta_{2}$, and black: $\theta_{\text {ref }}$. Since the control input is set to zero, the coupled pendula do not synchronize to the desired trajectory. Instead, the pendula synchronize in anti-phase and the oscillation frequency is almost twice the oscillation frequency of the desired trajectory. (Color figure online)

pendula will asymptotically synchronize to the desired reference trajectory.

To illustrate the above discussion, system (8)-(10) with input (14) is numerically integrated by using the parameter values mentioned above and the following initial conditions: $\theta_{1}(0)=-0.02[\mathrm{rad}], \theta_{2}(0)=0.018$ $[\mathrm{rad}]$, and the remaining initial conditions are all set to zero.

In a first numerical study, the control input $u(t)$, see Eq. (14), is set to zero. Obviously, for this case the oscillators do not synchronize to the desired synchronous solution, as shown in Fig. 3. Instead, the oscillators exhibit anti-phase synchronization and the frequency of the synchronous solutions is entirely determined by the intrinsic parameters of the uncontrolled system. In fact, as shown in the bottom panel of Fig. 3, the oscillation frequency of the pendula is almost twice the oscillation frequency of the reference trajectory $\theta_{\text {ref }}$.

In a second numerical study, the proposed input (14) is applied to the system of coupled pendula (8)-(10). The parameter values and initial conditions are exactly the same as those used in the first numerical study. As expected, when the open-loop control is applied, the

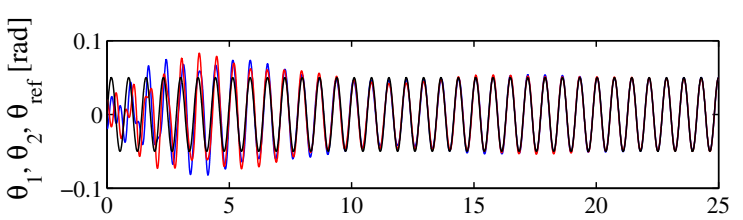

(a)

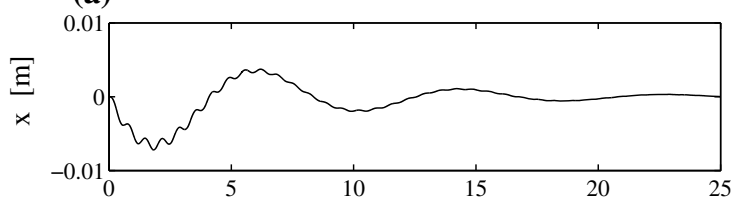

(b)

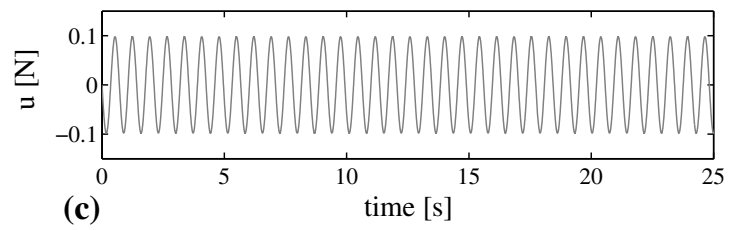

Fig. 4 Limit behavior of the controlled system (8)-(10), with control (14). Top: limit behavior of the coupled pendula. Blue line: $\theta_{1}$ [rad]. Red line: $\theta_{2}$ [rad], Black line: desired reference $\theta_{\text {red }}[\mathrm{rad}]$. Middle: horizontal displacement $x[\mathrm{~m}]$ of the coupling bar. Bottom: applied open-loop control. (Color figure online)

pendula synchronize to the desired reference trajectory, as shown in the top panel of Fig. 4. Furthermore, the coupling rigid bar comes to standstill once the pendula synchronize, as shown in the middle panel of Fig. 4. Finally, the applied control input (14) is depicted in the bottom panel of Fig. 4. Clearly, the proposed openloop synchronization strategy successfully induces the desired synchronous motion in the pendula.

\subsection{Generalized Huygens' system with feed-forward control}

Now, the analytic results presented in Sect. 4 are numerically illustrated. For that, system (39) is numerically integrated by using the following parameter values, which have been (partly) taken from the experimental platform presented in [35]: $\omega=13.2950$ [rad], $\xi=9 \times 10^{-3}[-], k=5[\mathrm{~N} / \mathrm{m}], \lambda=595.7699[-$ ], $m=0.210[\mathrm{~kg}], \kappa=37.10[\mathrm{~N} / \mathrm{m}], \mu=0.0515$ [-]. For these parameter values, it follows that the value of $H^{*}$, see Eq. (61), should be chosen as $H^{*}=1.1 \times 10^{-3}$. The initial conditions in the oscillators are $x_{1}(0)=$ $3 \times 10^{-3}[\mathrm{~m}], x_{2}(0)=[\mathrm{m}]$

First, the limit behavior of the uncontrolled system is investigated. The obtained results are shown in Fig. 5. Note that although the oscillators are released close 


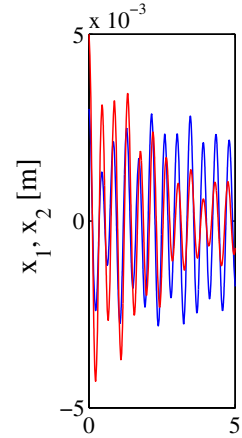

(a) time $[\mathrm{s}]$

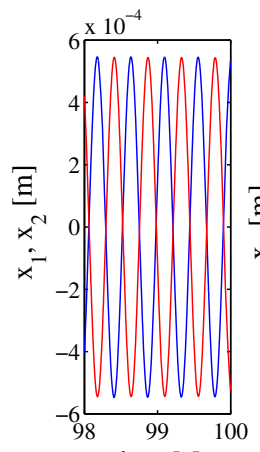

(b) time $[\mathrm{s}]$

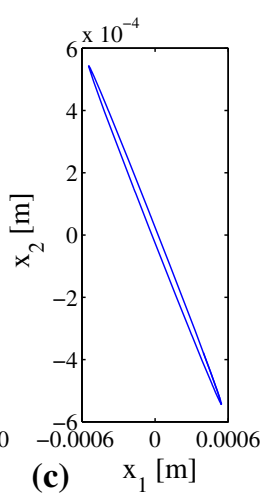

Fig. 5 Numerical results for the uncontrolled system. a Transient behavior. b Limit behavior. c Projection of the trajectories on the $\left(x_{1}, x_{2}\right)$-plane. The uncontrolled system has the tendency to synchronize in (almost) anti-phase and the amplitude, frequency, and phase of the solutions are entirely determined by the intrinsic parameters of the system

to in-phase, see Fig. 5a, after transients, the oscillators synchronize in (almost) anti-phase as shown in Fig. 5b, c. It should be noted that perfect anti-phase synchronization is not possible due to the coupling spring $k$, see Fig. 2 and model (39). In this case the amplitude, frequency, and phase of the periodic synchronous solution is completely determined by the intrinsic parameters of the system.

Then, in a second study, the control input (44) is applied to system (39). The synchronization objective is as given in (42) with $x_{\text {ref }}$ as given in (43) with $\eta=6 \times 10^{-3}$ and $\omega_{d}=\omega=13.2930$. For the considered parameter values, it is expected from Theorem 3 that the desired synchronous solution (42) exists and is locally asymptotically stable. The obtained results are shown in Fig. 6. Clearly, the oscillators synchronize to the desired reference signal $x_{\text {ref }}$, as shown in Fig. 6a, b. Furthermore, when the oscillators synchronize, the coupling bar comes to standstill as shown in Fig. 6c. Finally, the applied control input is shown in Fig. 6d.

These results nicely illustrate the good performance of the proposed feed-forward controller.

\subsection{On the robustness of the proposed controller}

Finally, we present a numerical study in order to illustrate the performance of the controller when there are parameter mismatches between the oscillators. In particular, the case where the oscillators have slightly different eigenfrequency is considered. Hence, system
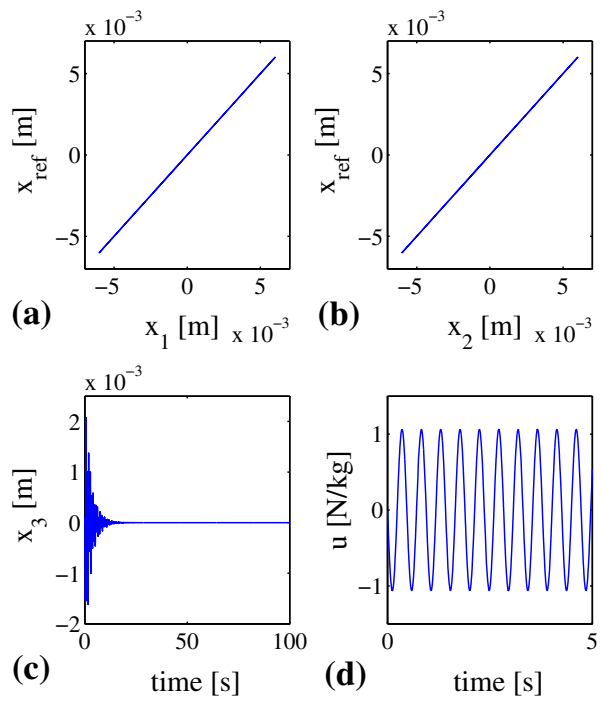

Fig. 6 Numerical results for the controlled system. Clearly, the controlled system performs as desired: the oscillators synchronize to the reference trajectory $x_{\text {ref }}$ as shown in $\mathbf{a}, \mathbf{b}$, and the coupling bar comes to standstill, as shown in $\mathbf{c}$. The feed-forward control applied to the coupling bar is shown in $\mathbf{d}$

(39) is again numerically integrated by using the parameter values used in Sect. 5.2, except that the stiffness coefficient $\kappa$ of oscillator 1 is assumed to have a $10 \%$ variation with respect to the nominal value. Therefore, the parameter $\kappa$ corresponding to oscillator 1 is $\kappa=38.9634[\mathrm{~N} / \mathrm{m}]$. Consequently, the eigenfrequency of oscillator 1 is $\omega=13.6213$ [rad], whereas the eigenfrequency of oscillator 2 coincides with the eigenfrequency of the reference trajectory, which is $w_{d}=13.2930$ [rad].

The obtained results are shown in Fig. 7 from which it is clear that due to the frequency mismatch, perfect tracking of the desired reference $x_{\text {ref }}$ is not possible, as shown in Fig. 7a, b. In particular, Fig. 7c shows that oscillator one has smaller amplitude (blue signal) than the reference signal (black signal), whereas the amplitude of the second oscillator (red signal) is larger than the amplitude of the reference signal. However, the oscillators are still synchronized in frequency to the reference trajectory. Furthermore, the coupling bar does not come to standstill, but rather it keeps oscillating with small amplitude. The reason is because, due to the parameter mismatch between the oscillators, the right-hand side of the third equation in (39) does not vanish. 

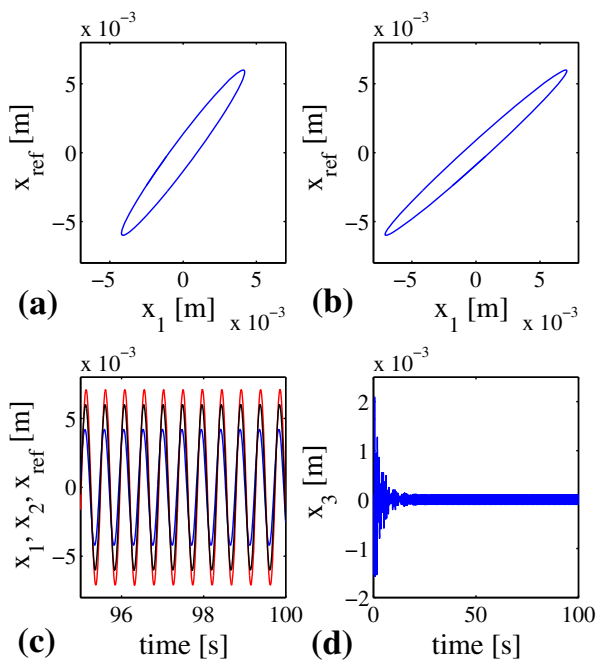

Fig. 7 Numerical results for the case of nonidentical oscillators

In summary, the proposed controller cannot induce perfect tracking of the reference trajectory when there are parameter mismatches. This, however, is not surprising since all the analysis has been conducted under the assumption of identical oscillators. Nevertheless, the results presented here suggest that, for nonidentical oscillators, the controller is able to induce at least frequency synchronization.

\section{Discussion and conclusions}

This work has presented a synchronization strategy based on a feed-forward controller for self-sustained oscillators interacting via Huygens' coupling. The proposed controller overcomes the natural tendency of the oscillators to synchronize and instead, enforces a desired synchronous solution.

Two particular cases have been considered, namely the classical Huygens' system of pendulum clocks and a pair of self-sustained mass-spring-damper oscillators. For both cases, the proposed feed-forward controller is able to induce the desired synchronous motion. This fact suggest that the proposed synchronization strategy may be applicable to a larger class of selfsustained oscillators.

Furthermore, a modification has been made to the classical Huygens' system. This modification, which consists in adding a linear coupling spring between the masses of the oscillators, is key since without this spring, it is impossible to induce the desired synchronous behavior, as discussed in Remarks 2 and 4.

On the other hand, a formal analysis of the controlled system has been conducted by using the Poincaré method of perturbation. The analysis has revealed that the proposed feed-forward controller may enforce the desired synchronous motion, provided that some intrinsic parameters of the pendula satisfy certain conditions, as discussed in Theorem 2. Furthermore, sufficient and necessary conditions that guarantee the asymptotic stability of the desired synchronous solution have also been provided.

At this point, it is worth mentioning that the proposed controller requires that the intrinsic parameters in the oscillators satisfy certain constraints. Specifically, it is required that the eigenfrequency of the oscillators should coincide with the frequency of the desired trajectory. This can be a limitation in the cases that the parameters of the system cannot be modified or chosen freely.

Also, it is important to stress the fact that, although we have presented a numerical study related to the robustness of the proposed controller against parameter mismatches, see Sect. 5.3, the analytic results presented in this work have been derived under the assumption of identical oscillators. It would be interesting to further analyze the robustness properties of this synchronization strategy. A first step on this direction may be to experimentally validate this results by using the experimental platform presented in [35].

Finally, it should be noted that the controller does not require to have any knowledge about the state of the oscillators, i.e., the controller can indeed be seen as an open-loop controller and the resulting synchronization may be referred to as open-loop synchronization. Note that open-loop synchronization-to synchronize two or more systems without using feedback-may find interesting applications. For example, in [37] it has been experimentally demonstrated that it is possible to synchronize several uncoupled Lorenz systems by applying a common clock-like signal to all of them. As experimentally demonstrated by the author of that reference, such scheme can be used for the encryption and recovery of low-frequency audio signals with random noise. Another interesting application of openloop synchronization is in power grids. For example, in [31], an open-loop synchronization technique for a power grid is presented. It is demonstrated that this open-loop technique has a faster dynamic response than 
a closed-loop technique and also the open-loop scheme can be easily customized for different grid scenarios, like highly imbalanced and distorted grid conditions.

\section{Compliance with ethical standards}

Conflict of interest The authors declare that they have no conflict of interest.

\section{References}

1. Bell, A.E.: The horologium oscillatorium of Christian Huygens. Nature 148, 245-248 (1941)

2. Huygens, C.: Oeuvres completes de Christiaan Huygens. In: Nijhoff, M. (ed.) Correspondance 1664-1665, vol. V. La Societe Hollandaise des Sciences, The Hague (1893)

3. Oud, W.T., Nijmeijer, H., Pogromsky, A.Y.: A Study of Huijgens' Synchronization: Experimental Results. Lecture Notes in Control and Information Science, vol. 336, pp. 191203. Springer, Heidelberg (2006)

4. Yang, J., Wang, Y., Yu, Y., Xiao, J., Wang, X.: Huygens' synchronization experiment revisited: luck or skill? Eur. J. Phys. 39, 1-14 (2018)

5. Nijmeijer, H., Pogromsky, A.Y.: Huygens' synchronization: a challenge. In: Leonov, G., Nijmeijer, H., Pogromsky, A.Y., Fradkov, A. (eds.) Dynamics and Control of Hybrid Mechanical Systems. World scientific, Singapore (2010)

6. Belykh, V.N., Pankratova, E.V.: Shilnikov chaos in oscillators with Huygens coupling. Int. J. Bifurc. Chaos 24, 1440007 (2014)

7. Belykh, V.N., Pankratova, E.V.: Chaotic dynamics of two Van der Pol-Duffing oscillators with Huygens coupling. Regul. Chaotic Dyn. 14, 274-284 (2010)

8. Bennett, M., Schatz, M.F., Rockwood, H., Wiesenfeld, K.: Huygens's clocks. Proc. Math. Phys. Eng. Sci. 458, 563-579 (2002)

9. Boda, S., Néda, Z., Tyukodi, B., Tunyagi, A.: The rhythm of coupled metronomes. Eur. Phys. J. B 86, 1-9 (2013)

10. Pantaleone, J.: Synchronization of metronomes. Am. J. Phys. 70, 992-1000 (2002)

11. Jovanovic, V., Koshkin, S.: Synchronization of Huygens' clocks and the Poincaré method. J. Sound Vib. 331, 28872900 (2012)

12. Dilao, R.: On the problem of synchronization of identical dynamical systems: the Huygens's clocks. In: Buttazzo, G., Frediani, A. (Ed.) Variational Analysis and Aerospace Engineering. Springer Optimization and Its Applications, vol. 33. Springer, New York (2009)

13. Dilao, R.: Antiphase and in-phase synchronization of nonlinear oscillators: the Huygens's clocks system. Chaos Interdiscip. J. Nonlinear Sci. 19, 023118 (2009)

14. Wiesenfeld, K., Borrero-Echeverry, D.: Huygens (and others) revisited. Chaos Interdiscip. J. Nonlinear Sci. 21, 047515 (2011)

15. Pena Ramirez, J., Aihara, K., Fey, R.H.B., Nijmeijer, H.: Further understanding of Huygens' coupled clocks: the effect of stiffness. Phys. D: Nonlinear Phenom. 270, 11-19 (2014)

16. Hoogeboom, F.N., Pogromsky, A.Y., Nijmeijer, H.: Huygens' inspired multi-pendulum setups: experiments and stability analysis. Chaos Interdiscip. J. Nonlinear Sci. 26, 116304 (2016)

17. Jia, J., Song, Z., Liu, W., Kurths, J., Xiao, J.: Experimental study of the triplet synchronization of coupled nonidentical mechanical metronomes. Sci. Rep. 5, 17008 (2015)

18. Song, Z., Wu, Y., Liu, W., Xiao, J.: Experimental study of the irrational phase synchronization of coupled nonidentical mechanical metronomes. PLoS ONE 10, 1-11 (2015)

19. Czolczynski, K., Perlikowski, P., Stefanski, A., Kapitaniak, T.: Synchronization of two nonidentical clocks: what Huygens was able to observe? In: Kyamakya, K., Halang, W., Mathis, W., Chedjou, J., Li, Z. (eds.) Selected Topics in Nonlinear Dynamics and Theoretical Electrical Engineering. Studies in Computational Intelligence, vol. 459. Springer, Berlin (2013)

20. Senator, M.: Synchronization of two coupled escapementdriven pendulum clocks. J. Sound Vib. 291, 566-603 (2006)

21. Czołczyński, K., Perlikowski, P., Stefanski, A., Kapitaniak, T.: Why two clocks synchronize: energy balance of the synchronized clocks. Chaos Interdiscip. J. Nonlinear Sci. 21, 023129 (2011)

22. Pena Ramirez, J., Olvera, L.A., Nijmeijer, H., Alvarez, J.: The sympathy of two pendulum clocks: beyond Huygens' observations. Sci. Rep. 6, 23580 (2016)

23. Martens, E.A., Thutupalli, S., Fourrière, A., Hallatschek, O.: Chimera states in mechanical oscillators network. Proc. Natl. Acad. Sci. 110, 10563-10567 (2013)

24. Willms, A.R., Kitanov, P.M., Langford, W.F.: Huygens' clocks revisited. R. Soc. Open Sci. 4, 1-33 (2017)

25. Kapitaniak, M., Czolczynski, K., Perlikowski, P., Stefanski, A., Kapitaniak, T.: Synchronization of clocks. Phys. Rep. 517, 1-70 (2012)

26. Kapitaniak, T., Czolczynski, K., Perlikowski, P., Stefanski, A.: Energy balance of two synchronized self-excited pendula with different masses. J. Theor. Appl. Mech. 50, 729-741 (2012)

27. Oud, W., Nijmeijer, H., Pogromsky, A.Y.: Experimental results on Huygens synchronization. IFAC Proc. Vol. 39, 113-118 (2006)

28. Oliveira, H.M., Melo, L.V.: Huygens synchronization of two clocks. Sci. Rep. 5, 11548 (2015)

29. Czolczynski, K., Perlikowski, P., Stefanski, A., Kapitaniak, T.: Huygens' odd sympathy experiment revisited. Int. J. Bifurc. Chaos 21, 2047-2056 (2011)

30. Pena Ramirez, J., Denasi, A., Rodriguez-Angeles, A., Alvarez Gallegos, J., Nijmeijer, H., Aihara, K.: Controlled synchronization: a Huygens' inspired approach. IFAC Proc. Vol. 47, 3098-3103 (2014)

31. Golestan, S., Vidal, A., Yepes, A.G., Guerrero, J.M., Vasquez, J.C., Doval-Gandoy, J.: A true open-loop synchronization technique. IEEE Trans. Ind. Inform. 12, 1093-1103 (2016)

32. Blekhman, I.I.: Synchronization in Science and Technology. ASME Press, New York (1988)

33. Blekhman, I.I.: Synchronization of Dynamic Systems. Nauka, Moscow (1971) 
34. Pena Ramirez, J., Nijmeijer, H.: The Poincaré method: a powerful tool for analyzing synchronization of coupled oscillators. Indagationes Mathematicae 48, 1-20 (2016)

35. Pena Ramirez, J., Fey, R.H.B., Nijmeijer, H.: Synchronization of weakly nonlinear oscillators with Huygens' coupling. Chaos Interdiscip. J. Nonlinear Sci. 23, 033118 (2013)

36. Pena Ramirez, J., Aihara, K., Fey, R.H.B., Nijmeijer, H.: Further understanding of Huygens' coupled clocks: the effect of stiffness. Phys. D: Nonlinear Phenom. 270, 11-19 (2014)
37. Nunez-Perez, R.: Atypical synchronization of multiple uncoupled chaotic circuits and its application in encryption. Ingenieria, Investigacion y Tecnologia 13, 489-502 (2012)

Publisher's Note Springer Nature remains neutral with regard to jurisdictional claims in published maps and institutional affiliations. 